\title{
Matrix Order and $W^{*}$-Algebras in the Operational Approach to Statistical Physical Systems
}

\author{
Gerd Wittstock \\ Universität des Saarlandes, D-6600 Saarbrücken, Federal Republic of Germany
}

\begin{abstract}
An important problem in the axiomatic approach to statistical physical systems is to characterize ordered vector spaces that are isomorphic to the predual of a $W^{*}$-algebra. Recent work of Werner has shown that the set of interactive neutral hereditary projection on a matrix ordered complete base norm space $V$ is order isomorphic to the lattice of projections of a $W^{*}$-algebra, called the matrix multiplier algebra. If there are sufficiently many of these projections, then $V$ is the predual of its matrix multiplier algebra. This mathematical conception is motivated by physics. The result shows that matrix order instead of merely partially order provides a setting in which an axiomatic approach to statistical physical systems may be studied. In this paper the discussion on the physical relevance of the conception of matrix order and interactive neutral hereditary projections is started.
\end{abstract}

\section{Introduction}

An important problem in the axiomatic approach to statistical physical system especially to axiomatic quantum mechanics is to characterize partially ordered vector spaces that are isomorphic to the predual of a $W^{*}$-algebra. Recent work of Werner [13] has shown that the set of interactive neutral hereditary projections (inh-projections) on a matrix ordered complete base norm space $V$ is isomorphic to the lattice of projections of a $W^{*}$-algebra, called the matrix multiplier algebra of $V$. If there are sufficiently many inh-projections on $V$, then $V$ is the predual of its matrix multiplier algebra. Werners mathematical conception is motivated by physics.

In this paper we want to explain the physical meaning of matrix order and interactive neutral hereditary projections. We use the formulation of the operational approach to the theory of statistical physical systems in terms of partially ordered vector spaces due to Davis and Lewis [5]. Matrix ordered spaces were introduced by Powers [10] and Choi and Effros [2] as the objects to which complete positive morphisms apply. We show that matrix order is physically 
motivated by a coupling process of a general statistical physical system I with a second special quantum system II. System II is described by the ordered space of trace class operators on a separable Hilbert space as usual in classical algebraic quantum mechanics. Interactive operations on system I are compatible under the coupling process with the pure state projections of system II. Neutral and hereditary projections describe the operation of ideal filters. The simple observable measuring the transition probability for a state under an interactive neutral hereditary projection is called an inh-projective unit. The assumption, that the inhprojective units separate the states implies that system I is described by a von Neumann algebra model.

Matrix order provides an intermediate stage between merely ordered spaces and $C^{*}$-algebras. The axioms are physically motivated. The author, being a mathematician, suggests to analyze the introduction of matrix order in the operational approach to statistical physical systems more carefully. Especially it is desirable to explain the matrix order of system I without assuming the conventional description on Hilbert space of the second system II. In an Appendix we give an alternative equivalent system of axioms for matrix order, suggesting an approach using several coupling processes. There is notion of a matrix convex set dual to the notion of a matrix ordered order unit space. There are other characterisations of $C^{*}$ - and $W^{*}$-algebras in terms of matrix order respectively $n$ order $(n=2,3,4, \ldots)$ : Choi and Effros [2] show that injective matrix ordered order unit spaces are injective $C^{*}$-algebras. In [16] injective $W^{*}$-algebras are characterized as dual matrix ordered order unit spaces which fulfill a matricial analogue of the Riesz separation property. In his thesis [12] Werner characterizes $C^{*}$-algebras in terms of 2-respectively 3-ordered order unit spaces. Along the lines of Connes work [4] Schmitt shows that 2-ordered homogeneous selfdual cones are standard representations of $W^{*}$-algebras [11].

\section{The Ordered Vector Space Approach}

Briefly, the operational approach of Davies and Lewis [5], Edwards [6] and others may be described as follows. The set of states of a statistical physical system is represented by a generating cone $V_{+}$of a real vector space $V_{+}-V_{+}$. For mathematical reasons we introduce the complexification $V=\left(V_{+}-V_{+}\right)$ $+i\left(V_{+}-V_{+}\right)$and an involution $\left(v_{1}+i v_{2}\right)^{*}:=v_{1}-i v_{2}$ for $v_{1}, v_{2} \in V_{+}-V_{+}$. $V_{h}:=V_{+}-V_{+}$is the hermitian part of $V$. Henceforth we call such a structure a $*_{-}$ ordered vector space. $V_{+}$has a base $K$, which represents the normalized states. $V_{h}$ is complete with respect to the base norm. We do not require that $V_{h}$ has the minimal decomposition property. The dual space $A_{h}=V_{h}^{d}$ is a complete order unit space with order unit $e$ defined for $\varphi_{1}, \varphi_{2} \in V_{+}$by $\left\langle\varphi_{1}-\varphi_{2}, e\right\rangle=\left\|\varphi_{1}\right\|-\left\|\varphi_{2}\right\|$. For $\varphi \in V_{+},\langle\varphi, e\rangle=\|\varphi\|$ is the strength of the state $\varphi . A=A_{h}+i A_{h}$ is the complexification of $A_{h}$. $A$ is a *-ordered vector space. The order interval $[0, e]=L \subset A_{+}$ is called the set of simple observables. An operation on the system is represented by a positive norm non increasing linear operator $T: V_{h} \rightarrow V_{h}$.

Special examples are the von Neuman algebra model: $A$ is a $W^{*}$-algebra, $V$ its predual. In the conventional Hilbert space approach for irreducible quantum 
systems $V=T(\mathscr{H})$, the trace class on some separable Hilbert space $\mathscr{H}, A=B(\mathscr{H})$, the set of all bounded operators on $\mathscr{H}$, with duality $\langle\varphi, x\rangle=\operatorname{Tr}\left(x^{*} \varphi\right)$. Our description of the operational approach is somewhat simplified. To include classical probability theory or the algebraic approach of Haag and Kastler one uses a more general framework.

\section{Matrix Order}

Spaces with an admissible cone as an abstract setting in which completely positive mapping and the extension property of Arveson may be studied were considered by Powers [10]. Choi and Effros [2] made substantial progress in the theory. They introduced the attractive name "matrix order". The theory is represented in [2] and the survey article [7]. We use the terminology and notation of the appendix to [7].

Given a *-vectorspace $V$ we denote by $M_{n}(V)=V \otimes M_{n}$ the *-vector space of $n \times n$ matrices $v=\left[v_{i j}\right]$ with entries $v_{i j} \in V$ and we let $M_{n}=M_{n}(\mathbb{C})$. If $\Phi: V \rightarrow W$ is complex linear we define the linear map $\Phi_{n}=\Phi \otimes \mathrm{id}_{n}: M_{n}(V) \rightarrow M_{n}(W)$ by $\Phi_{n}\left[v_{i j}\right]=\left[\Phi\left(v_{i j}\right)\right]$.

Definition. A *-ordered vector space is matrix ordered, if each $M_{n}(V), n \in \mathbb{N}$, is ordered by a cone $M_{n}(V)_{+}$with the following property:

(m) if $\gamma \in M_{m, n}$ is any $m \times n$ matrix of complex numbers,

$$
\gamma^{*} M_{m}(V)_{+} \gamma \subset M_{n}(V)_{+} .
$$

A linear map $\Phi: V \rightarrow W, V, W$ matrix ordered, is said to be completely positive if $\Phi_{n}$ is positive for all $n \in \mathbb{N}$. If $\Phi$ is bijective, and both $\Phi$ and $\Phi^{-1}$ are completely positive, we say that $\Phi$ is a matricial order isomorphism.

$C^{*}$ - and $W^{*}$-algebras, their duals or preduals and other related spaces as the selfdual cones of standard representations, their *-invariant subspaces, have a natural matrix order. $M_{n}$ itself is matrix ordered [one has $M_{k}\left(M_{n}\right) \cong M_{k n}$ ].

A map $\Phi: M_{m} \rightarrow M_{n}$ is completely positive iff there is a finite set $\gamma_{1}, \ldots, \gamma_{l} \in M_{m, n}$ of $m \times n$ matrices such that $\Phi(\alpha)=\sum \gamma_{\lambda}^{*} \alpha \gamma_{\lambda}$ (Choi [3]). Hence condition (m) is equivalent to

$\left(\mathrm{m}^{\prime}\right)$ if $\Phi: M_{m} \rightarrow M_{n}$ is completely positive, then

$$
\left(\mathrm{id}_{V} \otimes \Phi\right)\left(M_{m}(V)_{+}\right) \subset M_{n}(V)_{+} .
$$

$V$ is said to be a base norm matrix ordered space if $V_{h}$ is a base norm ordered vector space which is matrix ordered by closed cones $M_{n}(V)_{+} \quad$ in the natural product topology of $\left.M_{n}(V)\right]$. The dual space $A=V^{\delta}$ is matrix ordered by the dual cones, $e \otimes 1_{n}$ is an order unit in $M_{n}(A)_{+}$and $K_{n}=\left\{\varphi \in M_{n}(V)_{+} \mid\left\langle\varphi, e \otimes 1_{n}\right\rangle=1\right\}$ is the base for $M_{n}(V)_{+}$.

\section{Physical Motivation of Matrix Order}

We consider a general statistical physical system I represented by a base norm space $V_{\mathrm{I}}$ and a irreducible quantum system II, represented by $V_{\text {II }} \cdot V_{\text {II }}$ is isomorphic 
to the space $T(\mathscr{H})$ of trace class operators on a separabel Hilbert space $\mathscr{H}$. The coupled system IxII is represented by a base norm space $V_{\text {IxII }}$.

System II has a natural matrix order. Kraus [8], Lindblatt [9] and others discuss complete positivity of operations in the algebraic approach. We require that there are some special complete positive operations on system II. We consider a sequence of orthonormal pure states in system II, represented by an orthonormal system $\left(\xi_{n}\right)_{n=1}^{\infty}$ in $\mathscr{H}$ and denote $\mathscr{H}_{n}=\operatorname{span}\left\{\xi_{1}, \ldots, \xi_{n}\right\} \subset \mathscr{H}, q_{n}: \mathscr{H} \rightarrow \mathscr{H}_{n}$ the orthogonal projection. We identify $T\left(\mathscr{H}_{n}\right) \cong M_{n}$ (with trace norm). The maps $Q_{n}: T(\mathscr{H}) \ni \varphi \rightarrow q_{n} \varphi q_{n}^{*} \in M_{n}$ and $J_{n}: M_{n} \ni \alpha \rightarrow q_{n}^{*} \alpha q_{n} \in T(\mathscr{H})$ are completely positive norm non increasing. We impose the following coupling condition:

(c) For any norm non increasing completely positive map $\Phi: M_{m} \rightarrow M_{n}$ is $J_{n} \Phi Q_{m}$ an operation on system $I I$. For these operations there is an induced operation $\Phi_{\mathrm{I}}$ on the coupled system IxII. The correspondence $\Phi \rightarrow \Phi_{\mathrm{I}}$ is affine

$$
(\lambda \Phi+(1-\lambda) \Psi)_{\mathrm{I}}=\lambda \Phi_{\mathrm{I}}+(1-\lambda) \Psi_{\mathrm{I}}, \quad 0 \leqq \lambda \leqq 1
$$

and functorial

$$
(\Phi \Psi)_{\mathrm{I}}=\Phi_{\mathrm{I}} \Psi_{\mathrm{I}} .
$$

The subspace $\left(\mathrm{id}_{1}\right)_{\mathrm{I}}\left(V_{\mathrm{IxII}}\right)$ is isomorphic to $V_{\mathrm{I}}$.

We can interpret the last condition as follows. If we keep the second part of the coupled system in a fixed pure state the resulting subsystem is canonical isomorphic to system I. $Q_{1}=J_{1} \operatorname{id}_{1} Q_{1}$, id $d_{1}$ the identity mapping of $M_{1}=T\left(\mathscr{H}_{1}\right)$, is the projection on a pure state. By the functorial property the corresponding induced map $\left(\mathrm{id}_{1}\right)_{\mathrm{I}}$ is a projection. The image $\left(\mathrm{id}_{1}\right)_{\mathrm{I}}\left(V_{\mathrm{IxII}}\right)$ is a subsystem, where the second part is in a fixed pure state.

Now we use simple linear algebra to show that condition (c) equips $V_{\text {I }}$ with a matrix order. We denote $V_{n}=\left(\mathrm{id}_{n}\right)_{\mathrm{I}}\left(V_{\mathrm{IxII}}\right)$. $V_{1}$ is isomorphic with $V_{\mathrm{I}}$. The complex linear span of the set of norm non increasing completely positive linear maps from $M_{m}$ into $M_{n}$ is the set of all linear maps from $M_{n}$ into $M_{n}$. The affine functor $\Phi \rightarrow \Phi_{\mathrm{I}}$ has a unique linear extension to the set of all linear maps. We have a linear functor from the category of finite dimensional vectorspaces $M_{n}$ to the category of vector spaces $V_{n}(n \in \mathbb{N})$. Hence there is a natural isomorphism $V_{n} \cong V_{1} \otimes M_{n}$, $\Phi_{\mathrm{I}} \mid V_{n} \cong \mathrm{id}_{v_{1}} \otimes \Phi . V_{1}$ is order isomorphic to $V_{\mathrm{I}} \cdot M_{n}\left(V_{\mathrm{I}}\right)=V_{\mathrm{I}} \otimes M_{n} \cong V_{n}$ is ordered by the cone $M_{n}\left(V_{\mathrm{I}}\right)_{+} \cong V_{n+}:=V_{n} \cap\left(V_{\mathrm{I} x \mathrm{II}}\right)_{+}$. Let $\Phi: M_{m} \rightarrow M_{n}$ be completely positive, then $\Phi_{\mathrm{I}}\left(V_{m+}\right) \subset V_{n+}$ hence $\left(\mathrm{id}_{V_{\mathrm{I}}} \otimes \Phi\right)\left(M_{m}\left(V_{\mathrm{I}}\right)_{+}\right) \subset M_{n}\left(V_{\mathrm{I}}\right)_{+}$. The condition $\left(\mathrm{m}^{\prime}\right)$ for a matrix ordered space is fulfilled.

\section{Neutral Hereditary Projections}

A fundamental problem in any approach to statistical physical systems is to define the proper notion of "proposition" $p \in L$ and the corresponding projection $P$ of states. In the von Neumann algebra model the propositions are the extreme points of $L=[0, e]$. These are the projections (hermitian idempotents) of the algebra $A$. $P: \varphi \rightarrow p \cdot \varphi \cdot p$ is the corresponding projection of a state $\varphi$. The Cauchy-Schwarz inequality is the source for the rich structure of the projections in a von Neumann 
algebra. In the ordered space approach one needs some additional requirements. Alfsen and Shultz defined an analogue, called a $P$-projection. The alternative definition of a $P$-projection which is given by Theorem 2.5 of [1] reflects properties of physical filters used for "yes-no" measurements. We introduce a weaker notion, the neutral hereditary projections (nh-projections). We do not assume the existence of a quasi-complement and the requirement of smoothness is considerably reduced. On the other hand we consider matrix ordered spaces and interactive projections. Matrix order bears some rudiments of the CauchySchwarz inequality. Hence interactive nh-projections on a 2-ordered space are already $P$-projections ([13], Appendix).

Definition. A neutral hereditary projection (nh-projection) on a base norm space $V$ is a norm non increasing positive idempotent operator $P: V \rightarrow V$ with the following properties:

(n) if $\varphi \in V_{+}$and $\|P \varphi\|=\|\varphi\|$ then $P \varphi=\varphi$,

(h) if $x \in A_{+}$and $x \leqq P^{\delta} e$ then $P^{\delta} x=x$.

$p:=P^{\delta} e$ is called the corresponding nh-projective unit.

$P$ represents the operation of an ideal filter. The filtered states are invariant under the filter: $P^{2}=P$. If the strength of a state $\varphi$ is undiminished $\|P \varphi\|=\|\varphi\|$ then the filter is neutral to the state: $P \varphi=\varphi$. If the expected values of a simple observable $x$ are less than the strength of the filtered states: $\langle\varphi, x\rangle \leqq\|P \varphi\|$ for all states $\varphi$ then $x$ measures only the filtered states: $\langle P \varphi, x\rangle=\langle\varphi, x\rangle$ for all $\varphi$.

By condition (h) $\mathrm{im}_{+} P^{\delta}=\left\{P^{\delta} x \mid x \in A_{+}\right\}$is a hereditary cone. The image of $P^{\delta}$ is the order ideal generated by $p$. By condition (n) $\operatorname{im}_{+} P=\left\{\varphi \mid \varphi \in V_{+}, P \varphi=\varphi\right\}$ $=\operatorname{ker}_{+}(e-p)=\left\{\varphi \mid \varphi \in V_{+},\langle\varphi, e-p\rangle=0\right\}$. Hence a nh-projection $P$ is uniquely determined by its nh-projective unit $p$.

A nh-projective unit is an extreme point of $L=[0, e]$. This implies that in a von Neumann algebra the extreme points of $L$ are the nh-projective units and nhprojections are of the form $\varphi \rightarrow p \cdot \varphi \cdot p$ with $p \in e x L$.

\section{Interactive nh-Projections}

Complete positivity of an operation is a natural and simple condition. In general this assumption is too weak and leads only in the case of central projections to interesting results. Interactivity will describe the interaction of a nh-projection on the first system I with a pure state projection on the second system II. The notation will be the same as in Chap. 3. $J_{m} \varepsilon_{n n} Q_{m}$ is the projection on the $n$-th pure state, where $\varepsilon_{n n} \in M_{m}$ is the usual $n$-th matrix unit and $1 \leqq n \leqq m . E_{n}:=\left(\varepsilon_{n n}\right)_{\mathrm{I}}$ denotes the induced operation on the coupled system IxII. The functorial property of the correspondence $\Phi \rightarrow \Phi_{\mathrm{I}}$ implies that $E_{n}$ is a projection independent of $m$. Recall that the subspace $V_{1}=E_{1}\left(V_{\mathrm{IxII}}\right)$ is order isomorphic to $V_{\mathrm{I}}$. A nh-projection $P$ on $V_{\mathrm{I}}$ is called interactive if there is an operation $R$ on $V_{\text {IxII }}$ with the following properties:

$\left(\mathrm{id}_{n}\right)_{\mathrm{I}} R=R\left(\mathrm{id}_{n}\right)_{\mathrm{I}}$ is a nh-projection on $V_{n}(n \in \mathbb{N}), E_{1} R=R E_{1} \cong P$ regarding the isomorphism $V_{1} \cong V_{\mathrm{I}}$ and $E_{n} R=R E_{n}=E_{n}(n=2,3 \ldots)$.

The restriction $R \mid V_{n}(n \in \mathbb{N}$ ) is characterized by the following properties (cf. [13], Lemma 3.3). 
Definition. Let $V$ be a complete matrix ordered base norm space. $P$ a nh-projection on $V . P$ is called interactive (=inh-projection) if the diagonal matrix $\operatorname{diag}(p, e, \ldots, e)$ is a nh-projective unit on $M_{n}(V)$.

We denote the set of inh-projections by $P_{\infty}$ and the set of inh-projective units by $U_{\infty} . P_{\infty}$ is ordered by the usual order of projections : $P \leqq Q \Leftrightarrow P Q=Q P=P . U_{\infty}$ has the relative order of $A$. By Werner's result ([13], Theorem 5.1) $U_{\infty}$ and $P_{\infty}$ are order isomorphic to the lattice of all projections $P\left(A_{m}\right)$ of a $W^{*}$-algebra $A_{m}$, called the matrix multiplier algebra. The map $P\left(A_{m}\right) \rightarrow U_{\infty}$ has a unique $w^{*}$-continuous linear extension $A_{m} \rightarrow A$ which is a matricial order isomorphism. Hence $A_{m}$ is embedded in $A$ as a $w^{*}$-closed subspace which is the closed linear span of $U_{\infty} . A$ and $V$ are unit linked bimodules over $A_{m}$. For $P \in P_{\infty}$ and $p \in U_{\infty}$ we have the formulas $P \varphi=p \cdot \varphi \cdot p$ and $P^{\delta} x=p x p(\varphi \in V, x \in A)$. Moreover $V$ and $A$ are $A_{m^{-}}$ matrix ordered: $g^{*} \cdot M_{m}(V)_{+} \cdot g \subset M_{n}(V)_{+}$and $g^{*} M_{m}(A)_{+} g \subset M_{n}(A)_{+}$for all $m \times n$ matrices $g$ with entries in $A_{m}$. (A similar notion was defined by Powers, the algebraically admissible cones [10].)

The predual $\left(A_{m}\right)_{\delta}$ is matricial isomorphic to $V / A_{m}^{\perp}$ with the quotient order. The states $\varphi, \psi$ are equivalent modulo $A_{m}^{\perp}$ iff the strength $\|P \varphi\|=\|P \psi\|$ for all inhprojections $P$. The assumption that the inh-projective units separate the states

$\varphi, \psi \in V_{+}$and $\langle\psi, p\rangle=\langle\psi, p\rangle$ for all $p \in U_{\infty} \Rightarrow \varphi=\psi$, implies that $A_{m}^{\perp}=0$, hence $A_{m}=A$ and $V$ is the predual of $A_{m}$.

\section{Superselection Rules and Matrix Order of Classical Systems}

Recall that the order centre $\mathcal{O}(V)$ is the span of those linear operators $T$ on $V$ for which $T$ and $\mathrm{id}_{V}-T$ are positive [14]. If $P \in \mathcal{O}(V), P^{2}=P$, then $P$ is said to represent a superselection rule. Then $P$ and $P^{\prime}:=\mathrm{id}_{V}-P$ are nh-projections.

It is easy to see that an inh-projection $P$ is in the order centre of $V$ iff the corresponding inh-projective unit $p$ is in the centre of the matrix multiplier algebra. An inh-projection is always completely positive. In general complete positivity does not imply interactivity of a nh-projection.

Theorem. Let $V$ be a complete matrix ordered base norm space and let $P \in O(V)$, $P^{2}=P . P$ is an inh-projection iff $P$ and $P^{\prime}$ are completely positive.

Proof. We denote by $Q_{1}: \alpha \rightarrow \varepsilon_{11} \alpha \varepsilon_{11}$ the nh-projection on the first pure state and by $Q^{\prime}: \alpha \rightarrow\left(1_{n}-\varepsilon_{11}\right) \alpha\left(1-\varepsilon_{11}\right)$ the complementary nh-projection in $M_{n}$, where $\varepsilon_{i j}$ denotes the usual matrix units. $\operatorname{Id}_{V} \otimes Q$ and $\mathrm{id}_{V} \otimes Q^{\prime}$ are nh-projections on $M_{n}(V)$ ([13], Lemma 3.2). The nh-projections $P \otimes \mathrm{id}_{n}, P^{\prime} \otimes \mathrm{id}_{n}, \mathrm{id}_{V} \otimes Q$, and $\mathrm{id}_{V} \otimes Q^{\prime}$ commute, hence $P \otimes Q, P \otimes Q^{\prime}, P^{\prime} \otimes Q$, and $P^{\prime} \otimes Q^{\prime}$ are nh-projections ([13], Lemma 2.3). Since $\left(P \otimes \mathrm{id}_{n}\right)\left(P^{\prime} \otimes Q^{\prime}\right)=\left(P^{\prime} \otimes Q^{\prime}\right)\left(P \otimes \mathrm{id}_{n}\right)=0, R:=P \otimes \mathrm{id}_{n}+P^{\prime} \otimes Q^{\prime}$ is positive, $R^{2}=R \cdot R^{\delta}\left(e \otimes 1_{n}\right)=\left(P^{\delta} \otimes \mathrm{id}_{n}+P^{\prime \delta} \otimes Q^{\prime \delta}\right)\left(e \otimes 1_{n}\right)=p \otimes 1_{n}+p^{\prime} \otimes\left(1_{n}-\varepsilon_{11}\right)$ $\leqq e \otimes 1_{n}$ implies that $R$ is norm non increasing.

Let $x \in M_{n}(A)_{+}, x \leqq p \otimes 1_{n}+p^{\prime} \otimes\left(1_{n}-\varepsilon_{11}\right)$. Then

$$
\left(P^{\prime \delta} \otimes \mathrm{id}_{n}\right) x \leqq p^{\prime} \otimes\left(1_{n}-\varepsilon_{11}\right)=\left(P^{\prime \delta} \otimes Q^{\prime \delta}\right)\left(e \otimes 1_{n}\right) \text {. }
$$


We obtain $\left(P^{\prime \delta} \otimes Q^{\prime \delta}\right) x=\left(P^{\prime \delta} \otimes Q^{\prime \delta}\right)\left(P^{\prime \delta} \otimes \mathrm{id}_{n}\right) x=\left(P^{\prime \delta} \otimes \mathrm{id}_{n}\right) x=x-\left(P^{\delta} \otimes \mathrm{id}_{n}\right) x$. Hence $x=R^{\delta} x . R$ is hereditary.

Let $\varphi \in M_{n}(V)_{+}$and $\left\langle\varphi, e \otimes 1_{n}\right\rangle=\left\langle R \varphi, e \otimes 1_{n}\right\rangle$. Then

$$
\left\langle\varphi, p \otimes 1_{n}+p^{\prime} \otimes 1_{n}\right\rangle=\left\langle\varphi, p \otimes 1_{n}+p^{\prime} \otimes\left(1_{n}-\varepsilon_{11}\right)\right\rangle,
$$

hence

$$
\begin{aligned}
\left\langle\left(P^{\prime} \otimes \mathrm{id}_{n}\right) \varphi, e \otimes 1_{n}\right\rangle & =\left\langle\varphi, p^{\prime} \otimes 1_{n}\right\rangle=\left\langle\varphi, p^{\prime} \otimes\left(1_{n}-\varepsilon_{11}\right)\right\rangle \\
& =\left\langle\left(P^{\prime} \otimes Q^{\prime}\right)\left(P^{\prime} \otimes \mathrm{id}_{n}\right) \varphi, e \otimes 1_{n}\right\rangle .
\end{aligned}
$$

Since $P^{\prime} \otimes Q^{\prime}$ is neutral it follows that

$$
\left(P^{\prime} \otimes Q^{\prime}\right) \varphi=\left(P^{\prime} \otimes Q^{\prime}\right)\left(P^{\prime} \otimes \mathrm{id}_{n}\right) \varphi=\left(P^{\prime} \otimes \mathrm{id}_{n}\right) \varphi=\varphi-\left(P \otimes \mathrm{id}_{n}\right) \varphi
$$

and therefore $R \varphi=\varphi . R$ is neutral. $R$ is a nh-projection with nh-projectiv unit $\operatorname{diag}(p, e, \ldots, e)$. Hence $P$ is an inh-projection.

Let $V$ be a matrix ordered space. Since any $\alpha \in M_{n+}$ is a square: $\alpha=\gamma^{*} \gamma$, condition $(\mathrm{m})$ implies that

$$
V_{+} \otimes M_{n+} \subset M_{n}(V)_{+} \text {. }
$$

The dual $V^{\delta}$ is matrixordered by the dual cones, hence

$$
V_{+}^{\delta} \otimes\left(M_{n}\right)_{+}^{\delta} \subset\left(M_{n}(V)\right)_{+}^{\delta} .
$$

Notice that $\left(M_{n}\right)_{+}^{\delta}$ is order isomorphic to $M_{n+}$ by the usual duality $\langle\alpha, \beta\rangle=\operatorname{trace}\left(\alpha^{t} \beta\right)$ for $\alpha, \beta \in M_{n} . M_{n}(V)_{+}$is a tensor cone in $V \otimes M_{n}$ ([15], Definition 1.11).

A classical statistical physical system is represented by a complete base norm space $V$ which is a vector lattice. $V$ is called an AL-space. Theorem 3.1 of [15] implies that for a Banach lattice the closure of any tensor cone coincides with the closure of the projective cone $C_{p}$. Especially for any Banach lattice there is a unique matrix order with closed cones $M_{n}(V)_{+}=\overline{\mathrm{co}}\left(V_{+} \otimes M_{n+}\right)$, where co denotes the closed convex hull in natural product topology of $M_{n}(V)$. Hence for classical systems matrix order yields no additional structure. The notion of an inhprojection and a projection in the order center coincide in this case.

\section{Appendix. An Alternative Introduction of Matrix Order}

The formulas (6.1) and (6.2) resulting from matrix order have a simple physical meaning. Let $V$ be a $*$-ordered vector space representing a general statistical physical system. $M_{n}$ represents a finite quantum system called a $n$-level system. The basic assumption of the following approach is that the coupled system is represented by $M_{n}(V)=V \otimes M_{n}$ ordered by a cone $M_{n}(V)_{+} \cdot M_{n}(V)^{\delta}$ is isomorphic to $M_{n}\left(V^{\delta}\right)$. It is ordered by the dual cone $M_{n}(V)_{+}^{\delta}$. Let $\varphi \in V_{+}, \alpha \in M_{n+}$ be states. $\varphi \otimes \alpha$ represents the coupling of the states $\varphi$ and $\alpha$ without interaction. Hence it is an element of $M_{n}(V)_{+}$. (6.1) is fulfilled. Let $x \in V_{+}^{\delta}, \beta \in\left(M_{n}\right)_{+}^{\delta}$ be simple observables. Then $x \otimes \beta$ represents the product of $x$ and $\beta$ without interaction of the two systems and is an element of $M_{n}(V)_{+}^{\delta}$. Hence 6.2 is fulfilled. 
For a $n$-level system $M_{n}$ and a $m$-level system $M_{m}$ we assume as usual that the coupled system $M_{m}\left(M_{n}\right)$ is order isomorphic to $M_{m n}$ by the natural identification $M_{n} \otimes M_{m}=M_{m n}$. The map $j_{m, n}: M_{m}\left(M_{n}\right) \rightarrow M_{n}\left(M_{m}\right), j_{m, n}(\alpha \otimes \beta)=\beta \otimes \alpha$ is an order isomorphism. $\alpha \otimes \beta$ denotes the usual Kronecker product of the matrices $\alpha \in M_{n}$, $\beta \in M_{m}$.

The next assumption is that the coupling process is associative

(m0) $\quad M_{m}\left(M_{n}(V)\right)_{+}=M_{m n}(V)_{+} \quad$ (associative law)

and commutative

(m1) $\quad \mathrm{id}_{V} \otimes j_{m, n}\left(M_{m}\left(M_{n}(V)\right)_{+}\right)=M_{n}\left(M_{m}(V)\right)_{+} \quad$ (commutative law).

The formulas (6.1) and (6.2) motivated above read as follows

$(\mathrm{m} 2) \quad M_{n}(V)_{+} \otimes M_{m+} \subset M_{m}\left(M_{n}(V)\right)_{+} \quad$ (product of states)

and

$\left(\mathrm{m}^{\prime}\right) \quad \operatorname{id}_{V} \otimes \mathrm{id}_{n} \otimes \alpha\left(M_{m}\left(M_{n}\right)_{+}\right) \subset M_{n}(V)_{+} \quad$ for all $\quad \alpha \in M_{m+}^{\delta}$.

We call the conditions (m 2 ) and (m 3$)$ the tensor cone laws.

We replace condition $(\mathrm{m} 3)$ by

$\left(\mathrm{m}^{\prime}\right) \quad \mathrm{id}_{V} \otimes \alpha\left(M_{m}\left(M_{n}\right)_{+}\right) \subset M_{n}(V)_{+} \quad$ for all $\alpha \in M_{m+}^{\delta}$.

If $V$ is a locally convex space with closed cones $M_{n}(V)_{+}$then $\left(\mathrm{m} 3^{\prime}\right)$ is equivalent to condition (m 3$)$. In general ( $\left.\mathrm{m}^{\prime}\right)$ implies (m 3).

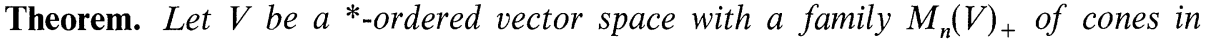
$M_{n}(V)(n \in \mathbb{N})$. Define $M_{m}\left(M_{n}(V)\right)_{+}$by $(\mathrm{m} 0)$. If the conditions $(\mathrm{m} 1),(\mathrm{m} 2),\left(\mathrm{m} 3^{\prime}\right)$ are satisfied, then $V$ is matrix ordered by the family $M_{n}(V)_{+}(n \in \mathbb{N})$. On the other hand any matrix ordered space $V$ fulfills $(\mathrm{m} 1),(\mathrm{m} 2)$, and $\left(\mathrm{m} 3^{\prime}\right)$.

Proof. Notice that $M_{n+}^{\delta}=M_{n+}$ by the usual duality $\langle\alpha, \beta\rangle=\operatorname{trace}\left(\beta^{t} \alpha\right)$ for $\alpha, \beta \in M_{n}$. $M_{n}$ has the canonical basis $\varepsilon_{i j}$ of matrix units. $M_{n^{2}}$ has the basis $\varepsilon_{i j} \otimes \varepsilon_{k l}$ $(i, j, k, l=1, \ldots, n)$. The matrix $\varepsilon=\sum \varepsilon_{i j} \otimes \varepsilon_{i j} \in M_{n^{2}}$ is positive since $\varepsilon^{2}=n \varepsilon$. The contraction $T^{n}: M_{n^{2}} \rightarrow \mathbb{C}$,

$$
T^{n}(\alpha)=\operatorname{trace}\left(\varepsilon^{t} \alpha\right)=\sum \alpha_{i j i j} \quad \text { for } \quad \alpha=\sum \alpha_{i j k l} \varepsilon_{i j} \otimes \varepsilon_{k l} \in M_{n^{2}}
$$

is a positive linear form. By condition $\left(\mathrm{m} 3^{\prime}\right)$ the map

$$
\mathrm{id}_{V} \otimes \mathrm{id}_{m} \otimes T^{n}: M_{n^{2}}\left(M_{m}(V)\right) \rightarrow M_{m}(V)
$$

is positive. Condition (m1) implies that

$$
\mathrm{id}_{V} \otimes T^{n} \otimes \mathrm{id}_{m}=\left(\mathrm{id}_{V} \otimes \mathrm{id}_{m} \otimes T^{n}\right)\left(\mathrm{id}_{V} \otimes j_{m, n^{2}}\right): M_{m}\left(M_{n^{2}}(V)\right) \rightarrow M_{m}(V)
$$

is "positive. Let $v=\sum v_{k l} \otimes \varepsilon_{k l} \in M_{n}(V)$. Then $v \otimes \varepsilon=\sum \sum v_{k l} \otimes \varepsilon_{k l} \otimes \varepsilon_{i j} \otimes \varepsilon_{i j}$ $\in M_{n}\left(M_{n^{2}}(V)\right)$. By definition of $T^{n}$ we have

$$
\left(\mathrm{id}_{V} \otimes T^{n} \otimes \mathrm{id}_{n}\right)(v \otimes \varepsilon)=\sum \sum v_{k l} \otimes T^{n}\left(\varepsilon_{k l} \otimes \varepsilon_{i j}\right) \otimes \varepsilon_{i j}=\sum v_{i j} \otimes \varepsilon_{i j}=v .
$$


By definition $(\mathrm{m} 0)$ and condition $(\mathrm{m} 2)$ we get

$$
M_{n}(V)_{+} \otimes M_{m}\left(M_{n}\right)_{+} \subset M_{m}\left(M_{n^{2}}(V)\right)_{+} .
$$

(7.1), (7.3), and (7.2) imply

$$
\left(\mathrm{id}_{V} \otimes T^{n} \otimes \mathrm{id}_{n}\right)\left(M_{n}(V)_{+} \otimes M_{n^{2}}\right)=M_{n}(V)_{+} .
$$

Let $\Phi: M_{n} \rightarrow M_{m}$ be a completely positive map. We have

$$
\left(\mathrm{id}_{V} \otimes \Phi\right)\left(\mathrm{id}_{V} \otimes T_{n} \otimes \mathrm{id}_{n}\right)=\left(\mathrm{id}_{V} \otimes T^{n} \otimes \mathrm{id}_{m}\right)\left(\mathrm{id}_{V} \otimes \mathrm{id}_{n} \otimes \mathrm{id}_{n} \otimes \Phi\right)
$$

on the vector space $M_{n}(V) \otimes M_{n^{2}}=V \otimes M_{n} \otimes M_{n} \otimes M_{n}$. Applying (7.4), (7.5), (7.3), and (7.1) we conclude that

$$
\begin{aligned}
\left(\mathrm{id}_{V} \otimes \Phi\right)\left(M_{n}(V)_{+}\right)= & \left(\mathrm{id}_{V} \otimes \Phi\right)\left(\mathrm{id}_{V} \otimes T^{n} \otimes \mathrm{id}_{n}\right)\left(M_{n}(V)_{+} \otimes M_{n}\left(M_{n}\right)_{+}\right) \\
= & \left(\mathrm{id}_{V} \otimes T^{n} \otimes \mathrm{id}_{m}\right)\left(\mathrm{id}_{V} \otimes \mathrm{id}_{n} \otimes \mathrm{id}_{n} \otimes \Phi\right)\left(M_{n}(V)_{+} \otimes M_{n}\left(M_{n}\right)_{+}\right) \\
& \subset\left(\mathrm{id}_{V} \otimes T^{n} \otimes \mathrm{id}_{m}\right)\left(M_{n}(V)_{+} \otimes M_{m}\left(M_{n}\right)_{+}\right) \\
& \subset\left(\mathrm{id}_{V} \otimes T^{n} \otimes \mathrm{id}_{m}\right)\left(M_{m}\left(M_{n^{2}}(V)_{+}\right)\right. \\
& \subset M_{m}(V)_{+} \cdot
\end{aligned}
$$

We have proved that condition $\left(\mathrm{m}^{\prime}\right)$ of Chap. 2 is fulfilled.

Let $V$ be a matrix ordered space. Then the spaces $M_{n}(V)$ are matrix ordered by the cones $M_{m}\left(M_{n}(V)\right)_{+}:=M_{m n}(V)_{+}$. The formulas (6.1), (6.2) imply (m2) and (m $\left.3^{\prime}\right)$. There is a permutation matrix $\pi \in M_{m n}$ such that $j_{m, n}(\alpha \otimes \beta)=\pi^{*}(\alpha \otimes \beta) \pi$. Hence condition (m1) is satisfied.

\section{References}

1. Alfsen, E.M., Shultz, F.W.: Non-commutative spectral theory for affine function spaces on convex sets. Memoir Am. Math. Soc. 172 (1976)

2. Choi, Effros, E.G.: Injectivity and operator spaces. J. Funct. Anal. 24, 156-209 (1977)

3. Choi, M.D.: Completely positive maps on complex matrices. Linear Algebra Appl. 10, 285-290 (1975)

4. Connes, A.: Charactérisation des espaces vectoriels ordonnés sous-jacent aux algèbres de von Neumann. Ann. Inst. Fourier Grenoble 24, 121-155 (1974)

5. Davies, E.B., Lewis, J.T.: An operational approach to quantum probability. Commun. Math. Phys. 17, 239-260 (1970)

6. Edwards, C.M. : Classes of operations in quantum theory. Commun. Math. Phys. 20, 26-56 (1971)

7. Effros, E.G.: Aspects of non-commutative order. In: $C^{*}$-algebras and applications to physics. Lecture notes in mathematics $\mathbf{6 5 0}, 1-40$ (1978)

8. Kraus, K.: Operations and effects in the Hilbert space formulation of quantum theory. In: Foundations of quantum mechanics and ordered linear spaces. Lecture notes in physics 29, 206-229 (1974)

9. Lindblatt, G.: On the generator of quantum dynamical semigroups. Commun. Math. Phys. 48, 119-130 (1975)

10. Powers, R.T.: Selfadjoint algebras of unbounded operators. II. Transact. Am. Math. Soc. 187, 261-293 (1974)

11. Schmitt, L.: Charakterisierung von $W^{*}$-Algebren durch autopolare 2-geordnete, diagonalhomogene, 2-positive Kegelpaare. Diplomarbeit, Universität des Saarlandes (1979)

12. Werner, K.H.: Charakterisierung von $C^{*}$-Algebren durch $p$-Projektionen auf matrix-n-geordneten Räumen. Dissertation, Universität des Saarlandes (1979) 
13. Werner, K.H.: A characterisation of $C^{*}$-algebras by nh-projections on matrix ordered spaces. (To appear)

14. Wils, W.: The ideal center of partially ordered vector spaces. Acta Math. 127, 41-79 (1971)

15. Wittstock, G.: Ordered normed tensor products. In: Foundations of quantum mechanics and ordered linear spaces. Lecture notes in physics 29, 67-84 (1974)

16. Wittstock, G.: Ein operatorwertiger Hahn-Banach Satz. (To appear in J. Funct. Anal.)

Communicated by H. Araki

Received November 5, 1979 